\title{
Exemplifying Conflict Resolution in Multi-Objective Smart Micro-Grids
}

\author{
Sylvain Frey* ${ }^{* \dagger}$, Ada Diaconescu*, David Menga ${ }^{\dagger}$ and Isabelle Demeure* \\ *Télécom ParisTech, CNRS LTCI, Paris, France. Email: \{first $\}.\{$ last $\} @$ telecom-paristech.fr \\ ${ }^{\dagger}$ EDF R\&D, Clamart, France. Email: $\{$ first $\} .\{$ last $\} @$ edf.fr
}

\section{Motivation}

Distributed autonomic management systems following contradictory objectives raise difficult design challenges [2]. We proposed a generic architecture to address this concern and exemplified it via manager integration solutions for multiobjective micro-grids (low-tension networks of the size of a district [4]) [3]. This demo showcases some of these sample implementations via the MisTiGriD simulation platform with the aim of inspiring designers facing similar challenges.

Power management in electrical grids, i.e. balancing productions and consumptions, is a primary concern. At the same time, in residential micro-grids, end-user objectives such as comfort or domestic usages directly conflict with power control goals. For instance, heating during cold winter nights would generally conflict with a consumption limitation goal.

A viable approach for handling conflicts in autonomic systems consists in introducing conflict resolution facilities in the management layer [2]. Such solutions allow heterogeneous managers to integrate with each other by negotiating conflicting goals. In the case of micro-grids, integration relies on power management organisations [3] where usage goals and power management goals are negotiated via variable organisational patterns [1], [3]. The resulting system behaviour may sacrifice some lower-priority objectives in order to fulfil more important ones, or find a good-enough trade-of, depending on changing environmental conditions, variable manageable resources and dynamic user specifications.

MisTiGriD was conceived in order to showcase such conflict resolution mechanisms. This demonstrator is based on a simulation of a district micro-grid, providing the experimenter with partial control such as switching appliances on and off or controlling atmosphere temperature. MisTiGriD also allows the experimenter to set variable, conflicting goals to simulated autonomic systems, for instance, "comfort" goals for smart heaters or a maximum consumption for the district grid. Thus, the experimenter can run a variety of scenarios and explore the capabilities and the limits of the management system, and in particular the way in which conflicts are solved by managers.

\section{Simulation FEATURES}

\section{A. Temperature}

MisTiGriD features several types of thermic objects that model systems with a temperature: rooms, heaters (in rooms)

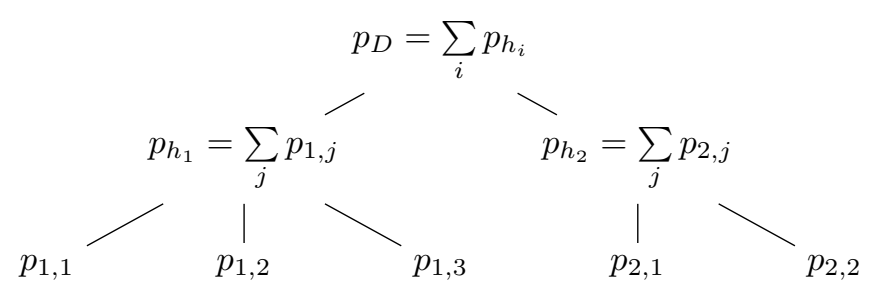

Fig. 1. Sample district grid: district aggregator $D$ and 2 houses $h_{1}$ and $h_{2}$ with 3 and 2 appliances repectively.

and the outside atmosphere. The atmosphere is considered a "thermal reservoir": its temperature is not influenced by other thermic objects, and the experimenter can set it to arbitrary values at run-time to simulate variable weather conditions. For non-atmosphere thermic objects, MisTiGriD models temperature exchanges via Fourier's law of heat transfer [5].

\section{B. Electric network}

The electric network in MisTiGriD is a tree of depth 2 (cf. Fig. 1). Domestic appliances are the leaves of the tree, their prosumptions ${ }^{1}$ being aggregated by their parent node at the house level. House aggregators are in turn the children of the root of the tree: the district aggregator. Each aggregator $\mathrm{Agg}$ (either house or district) is equivalent to a prosumer with a prosumption $p_{A g g}$ equal to the sum of prosumptions of its children $C$ (appliances or houses, respectively): $p_{\text {Agg }}=\sum_{\text {child } \in C} p_{\text {child }}$.

\section{Appliances}

MisTiGriD simulates the following appliances:

Lamps: model consumers with a constant consumption and an on/off behaviour. A wide range of appliances falls into this category, in addition to lightings, e.g. TV sets, hoovers, microwaves, kettles, computers may indeed be modelled as "lamps", each with their specific consumption level and typical period of use. In the proposed scenarios, all lamps consume 100W maximum, yet this parameter is easily configurable.

Heaters: thermic objects and consumers which convert electric power into heat. When switched on, a heater consumes a constant amount of power and raises its internal temperature to a high level (typically, $200 \mathrm{~W}$ for $50^{\circ} \mathrm{C}$ in the simulation),

\footnotetext{
1 either production (positive prosumption) or consumption (negative prosumption); the associated noun is prosumer.
} 
heating the room around it. When switched off, heater temperature progressively converges to its room's temperature. The cycle of heating/idle cycle is determined by the heater's autonomic manager, described in section III.

Others: MisTiGriD also features other prosumers not shown here, notably, batteries and producers such as solar panels.

\section{Autonomic ManAGEment}

There are three types of managers in the micro-grids: one district power manager, several house managers, and several appliance managers. House and district power objectives are maximum consumption levels that the experimenter can set to arbitrary values. Power managers at the house and district granularity try to maintain the corresponding aggregated consumption levels $-p_{h}$ and $p_{D}$ - below this threshold. Power manager achieve this goal by rescheduling prosumers in their scope (i.e. appliances in houses and houses in the district, respectively) [3]. This rescheduling may involve consumption reduction (see lamp and house behaviour below) or consumption shifting (see heater behaviour below).

Appliance managers follow a simple management objective, either "comfort" or "eco", implemented as follows:

Lamp behaviour: a lamp in "comfort" mode always consumes its maximum power (100W here). In case it is set to "eco", the manager accepts rescheduling by reducing its consumption (30W here).

Heater behaviour: a heater in "comfort" mode always maintains its minimum temperature objective (typically $22^{\circ} \mathrm{C}$ ). In case it is in "eco" mode, the manager accepts rescheduling by tolerating a temporary drop to $20^{\circ} \mathrm{C}$, shifting its heating cycle by a short period of time.

House power managers behaviour: when asked by the district power manager to reduce consumption, they lower their maximum consumption objective (by $600 \mathrm{~W}$ in the scenarios below). In turn, this change may trigger consumption reduction by local appliances.

\section{EXAMPLE SCENARIOS}

Fig. 2 depicts three possible scenarios in terms of the house prosumption objective (red), lamp consumption (blue) and total house consumption (lamps + heaters, green).

Scenario 1: the house consumption objective is constant and heaters are all in "eco" mode. When a set of "comfort" lamp consumptions are triggered around 100s, heaters compensate by rescheduling themselves and the house goal is not overshoot.

Scenario 2: only "eco" heaters are consuming, and the house consumption goal is reduced (either the experimenter changed the goal manually, or the district power manager issued a consumption reduction order). As a consequence, heaters reschedule themselves so as to fit in this new constraint as much as possible.

Scenario 3: same as scenario 1, but this time with heaters in "comfort" mode and a cold wave simulated from 800s. Since heaters are not allowed to reschedule themselves as they did in the previous scenarios, the house consumption goal is largely overshoot. This is what the user wanted, since she explicitly favoured comfort over energy economies.

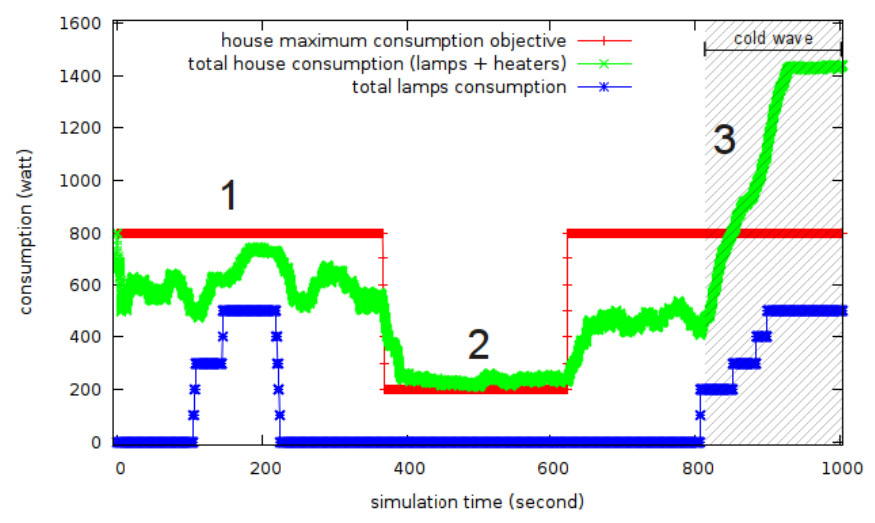

Fig. 2. House scenarios.

Fig. 3 shows a district of 8 houses being asked for a progressive consumption reduction (red maximum consumption objective being lowered manually by the experimenter). The district power manager orders the houses to reduce their consumption successively, so as to lower district consumption (in green). In turn the houses adapt their own consumption internally, consequently decreasing global consumption, until no more economies are possible (around 800s).

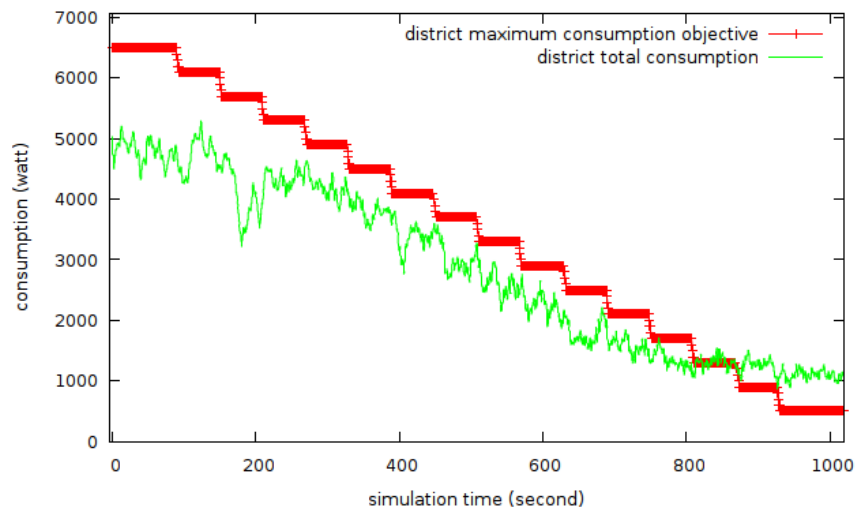

Fig. 3. District scenario.

\section{REFERENCES}

[1] S. Frey, A. Diaconescu, and I. Demeure. Architectural integration patterns for autonomic management systems. 9th IEEE International Conference and Workshops on the Engineering of Autonomic and Autonomous Systems (EASe), 2012.

[2] S. Frey, A. Diaconescu, D. Menga, and I. Demeure. Towards a reference model for multi-goal, highly-distributed and dynamic autonomic systems. 10th International Conference on Autonomic Computing (ICAC), 2013.

[3] S. Frey, A. Diaconescu, D. Menga, and I. Demeure. A holonic control architecture for a heterogeneous multi-objective micro smart grid. 7th IEEE International Conference on Self-Adaptive and Self-Organizing Systems (SASO), 2013 (to appear).

[4] S. Frey, F. Huguet, C. Mivielle, D. Menga, A. Diaconescu, and I. Demeure. Scenarios for an autonomic micro smart grid. 1st International Conference on Smart Grids and Green IT Systems (SmartGreens), 2012.

[5] http://en.wikipedia.org/wiki/Newton's_law_of_cooling. 